\title{
Docosahexaenoic acid inhibits the growth of hormone-dependent prostate cancer cells by promoting the degradation of the androgen receptor
}

\author{
ZHIMEI HU ${ }^{1}$, HAIXIA QI $^{1 *}$, RUIXUE ZHANG ${ }^{1 *}$, KUN ZHANG $^{1 *}$, ZHEMIN SHI $^{1}$, \\ YANAN CHANG $^{1}$, LINFENG CHEN ${ }^{2}$, MOHSEN ESMAEILI $^{3}$, ARIA BANIAHMAD ${ }^{3}$ and WEI HONG ${ }^{1}$ \\ ${ }^{1}$ Department of Histology and Embryology, School of Basic Medical Sciences, Tianjin Medical University, \\ Tianjin 300070, P.R. China; ${ }^{2}$ Department of Medical Oncology, Harvard Medical School, Dana Farber Cancer Institute, \\ Boston, MA 02115, USA; ${ }^{3}$ Institute of Human Genetics, Jena University Hospital, Jena D-07740, Germany
}

Received July 9, 2014; Accepted March 19, 2015

DOI: $10.3892 / \mathrm{mmr} .2015 .3813$

\begin{abstract}
Epidemiological and preclinical data have demonstrated the preventative effects of $\omega-3$ polyunsaturated fatty acids, including docosahexaenoic acid (DHA), on prostate cancer. However, there are inconsistencies in these previous studies and the underlying mechanisms remain to be elucidated. In the present study, the androgen receptor (AR), which is a transcription factor involved in cell proliferation and prostate carcinogenesis, was identified as a target of DHA. It was revealed that DHA inhibited hormone-dependent growth of LNCaP prostate cancer cells. Reverse transcription-quantitative polymerase chain reaction analysis revealed that treatment with DHA caused no alteration in the transcribed mRNA expression levels of the AR gene. However, immunoblotting revealed that this treatment reduces the protein expression level of the AR. The androgen-induced genes were subsequently repressed by treatment with DHA. It was demonstrated that DHA exhibits no effect on the translation process of the AR, however, it promotes the proteasome-mediated degradation of the AR. Therefore,
\end{abstract}

Correspondence to: Dr Wei Hong, Department of Histology and Embryology, School of Basic Medical Sciences, Tianjin Medical University, 22 Qixiangtai Road, Tianjin 300070, P.R. China

E-mail: hongwei@tijmu.edu.cn

Professor Aria Baniahmad, Institute of Human Genetics, Jena University Hospital, 10 Kollegiengasse, D-Jena 07740, Germany

E-mail: aria.baniahmad@med.uni-jena.de

*Contributed equally

Abbreviations: PUFAs, polyunsaturated fatty acids; DHA, docosahexaenoic acid; AR, androgen receptor; RT-qPCR, reverse transcription-quantitative polymerase chain reaction; MTT, 3, (4,5-dimethylthiazol-2-yl) 2,5-diphenyltetrazoliumbromide; CHX, cycloheximide

Key words: docosahexaenoic acid, prostate cancer, androgen receptor the present study provided a novel mechanism by which DHA exhibits an inhibitory effect on growth of prostate cancer cells.

\section{Introduction}

Prostate cancer is the most common type of cancer among males in Western countries (1). Although the initial cause of the onset of prostate cancer remains to be elucidated, previous studies have demonstrated potential links to dietary habits and fat intake. For example, a controlled case study provides evidence of a positive correlation between dietary fat and mortality from prostate cancer (2-5). The dietary intake of essential fatty acids, including $\omega-3$ and $\omega-6$ polyunsaturated fatty acids (PUFAs), is crucial for several cellular processes, including cell proliferation and differentiation (6). A number of previous studies have demonstrated that PUFAs are important in promoting or inhibiting several types of tumor, including hormone-responsive prostate tumors (7-9).

The contribution of $\omega-3$ and $\omega-6$ PUFAs to prostate carcinogenesis has gained considerable importance in previous years. It has been reported by previous in vitro and in vivo studies that $\omega-3$ PUFAs, including docosahexaenoic acid (DHA) and eicosapentaenoic acid (EPA), can repress the development and progression of prostate cancer, whereas $\omega-6$ PUFAs promote the growth of prostate cancer (9-12). In addition, epidemiological studies demonstrated that males who consumed large quantities of fish have a lower risk of prostate cancer and those who eat low quantities of seafood were associated with an increased prostate cancer risk, suggesting that there is an inverse correlation between diets rich in $\omega-3$ PUFAs and the incidence of prostate cancer (13-15). Therefore, the $\omega-3$ PUFAs contained in fish oil and other dietary factors may be beneficial for prostate cancer chemoprevention. However, the association between $\omega-3$ PUFAs and the progression from hormone dependency to hormone independency, and the mechanisms by which they may be involved in mediating their effects on androgen dependence remain to be elucidated.

The tumor-suppressive effects of $\omega-3$ PUFAs are hypothesized to be partly due to the modulation of signal transduction pathways (16-18). Androgens are important in the development 
and progression of prostate cancer (19). Androgens function via binding to the androgen receptor (AR), which is a ligand-dependent transcription factor of the nuclear hormone receptor superfamily. Therefore, AR is critical in the development of prostate cancer (19). Several previous studies have reported that the overexpression of AR is characteristic of prostate cancer that progresses to hormone independency (20-23). For instance, LNCaP clones, which progressed to hormone independency demonstrated increased protein expression levels of the AR, compared with their hormone-dependent syngenic clones. Exposure to $\omega-3$ PUFAs caused a significant effect on suppressing the androgen deprivation-induced expression of the AR (24).

The LNCaP cell line is an androgen-responsive prostate cancer cell line expressing the AR and a number of androgen-inducible genes, including prostate-specific antigen (PSA). The present study aimed to investigate whether treatment with DHA impedes the growth of hormone-responsive LNCaP cells, and whether the effect of DHA is associated with changes in the androgen receptor and androgen-regulated genes.

\section{Materials and methods}

Cell lines. All cells types used in the present study were obtained from the American Type Culture Collection (Rockville, MD, USA) and maintained in RPMI-1640 medium (Life Technologies, Grand Island, NY, USA), supplemented with $10 \%$ fetal bovine serum (FBS; Life Technologies), at $37^{\circ} \mathrm{C}$ and $5 \% \mathrm{CO}_{2}$, until reaching $70 \%$ confluence. The cells were subsequently treated with DHA (Sigma-Aldrich, St. Louis, MO, USA) dissolved in ethanol at the designated concentrations and for the indicated duration. For the AR stability experiment, the cells were treated with either $50 \mu \mathrm{g} / \mathrm{ml}$ of the protein synthesis inhibitor, cycloheximide (CHX; Sigma-Aldrich) for the indicated duration or $25 \mu \mathrm{M}$ proteasome inhibitor, MG132 (Sigma-Aldrich) for $24 \mathrm{~h}$ prior to harvesting.

Cell proliferation assay and PSA quantification. Cell growth was assessed by 3,(4,5-dimethylthiazol-2-yl)2,5-diphenyltetrazoliumbromide (Sigma-Aldrich) dye conversion, according to the manufacturer's instructions. Briefly, the cells were seeded $\left(5 \times 10^{3} /\right.$ well) into a 96 -well flat bottom plate and were treated with $0.4 \%$ trypan blue staining (Sigma-Aldrich). The cells were grown in different treatment conditions and cell growth was subsequently assessed following the indicated duration of continuous treatment. The number of viable cells was counted using a hemocytometer (XBK25; Qiujing Instrument, Shanghai, China) under a light microscope (x20 magnification; CKX31; Olympus, Tokyo, Japan).

The LNCaP cells were seeded at $3 \times 10^{4}$ cells/well in 24-well plates. Following culturing for $48 \mathrm{~h}$, the cells were treated with serum-free medium for $24 \mathrm{~h}$ and subsequently incubated in medium, containing $10 \%$ charcoal-stripped serum (Life Technologies) with indicated concentrations of DHA, in the absence or presence of $1 \mathrm{nM}$ R1881 (Perkin Elmer Life Sciences, Waltham, MA, USA). Following treatment for 5 days, the culture medium was collected for measuring the total protein expression levels of PSA, using the PSA Human kit (Abcam, Cambridge, MA, USA). The expression levels of PSA in the culture medium were normalized to the cell number.
Immunoblot and reverse transcription-quantitative polymerase chain reaction $(R T-q P C R)$. The cells were harvested and analyzed by immunoblotting, as previously described (25). The AR (\#3202) and GAPDH (D16H11; \#5174) antibodies were purchased from Cell Signaling Technology, Inc. (Danvers, MA, USA). For RT-qPCR analysis, the cells were suspended in $1 \mathrm{ml}$ TRIzol reagent (Life Technologies) and the total RNA was extracted, followed by cDNA synthesis as described previously (25). The RNA was amplified by RT-qPCR, performed with an SYBR Green Master Mix (Takara Biotechnology, Inc., Dalian, China) on a LightCycler ${ }^{\circledR} 96$ Real-Time PCR System (Roche, Mannheim, Germany). The cycling conditions were as follows: Initial denaturation at $95^{\circ} \mathrm{C}$ for $15 \mathrm{~min}$, followed by 40 cycles of $95^{\circ} \mathrm{C}$ for $5 \mathrm{sec}$ and $60^{\circ} \mathrm{C}$ for $30 \mathrm{sec}$. $\beta$-actin was used as the reference gene and the relative quantification comparative CT method was used. The primer sequences used are as follows: Forward 5'-GATGCTGTGAAGGTCATGGA-3' and reverse 5'-TGGAGGTCCACACACTGAAG-3' for PSA; forward 5'-TTGACTGCCACTTCCTCG-3' and reverse 5'-CATCCTTCGCCGACATGG-3' for ODC1; forward 5'-CTGGTGGCTGATAGGGGAT-3' and reverse 5'-GTCTGCCCTCATTTGTCGAT-3' for TMPRSS2; forward 5'-TCCCTCGAATGCAACTCTCT-3' and reverse 5'-GCCACATCTCTGCAGTCAAA-3' for FKBP51; and forward 5'-GCCAAGAACCTCAAGCTCAC-3' and reverse 5'-AGAAGGCCTCCTCTTTCAGG-3' for NKX3-1.

Statistical analysis. All data are presented as the mean \pm standard deviation. Data that followed a normal distribution were analyzed using Student's t-test or the one-way analysis of variance test for comparisons between two groups. Dunn's method was used for multiple comparisons. $\mathrm{P}<0.05$ was considered to indicate a statistically significant difference. All statistical values were calculated using SPSS software, version 19.0 (IBM SPSS, Armonk, NY, USA).

\section{Results}

DHA inhibits the growth of LNCaP cells. DHA has been demonstrated to suppress the growth of AR-positive, hormone-dependent LNCaP cells. The present study examined the efficacy of DHA on LNCaP cells, under conditions of hormone presence (in the presence of FBS), similar to the conditions in patients undergoing androgen-dependent carcinogenesis of prostate cancer. Firstly, increasing concentrations up to $100 \mu \mathrm{M}$ DHA were selected to treat the LNCaP cells for $6 \mathrm{~h}$, to assess whether DHA has a toxic effect. Trypan blue staining revealed no difference in the cells treated with DHA compared with the control cells (Fig. 1A), indicating that the concentrations of DHA used in the present study caused no toxic effect on LNCaP cells. As shown in Fig. 1B, when LNCaP cells growing in complete FBS were treated with DHA, there was decreased cell growth in a dose-dependent manner. In addition, treatment with $50 \mu \mathrm{M}$ DHA for varying durations on the LNCaP cells demonstrated a time-dependent suppression of cell growth (Fig. 1C). However, DHA-treated AR-negative PC3 and DU145 prostate cancer cells exhibited no response (Fig. 1D and E). This data suggested that AR potentiates the inhibitory effect of DHA on the growth of LNCaP cells when compared with those without the AR. 
A

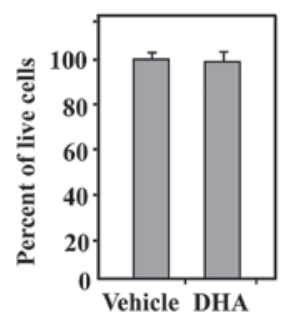

C

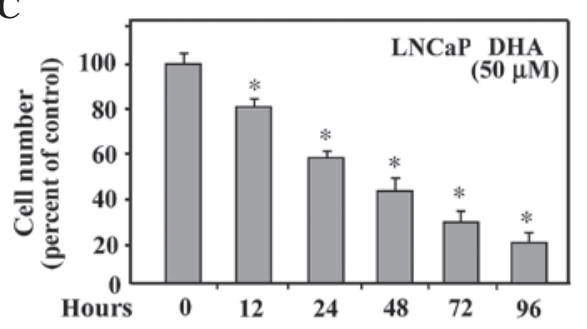

B

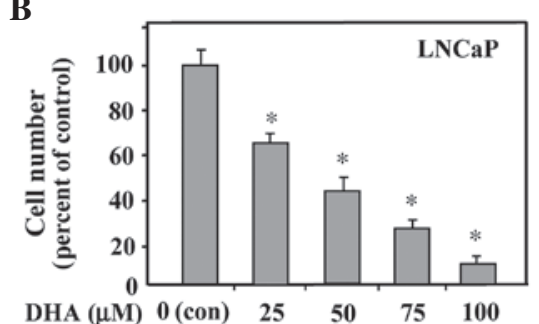

D

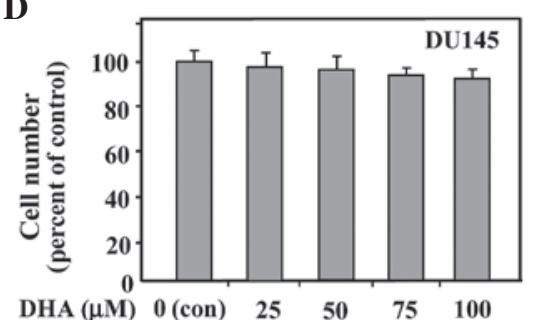

$\mathbf{E}$

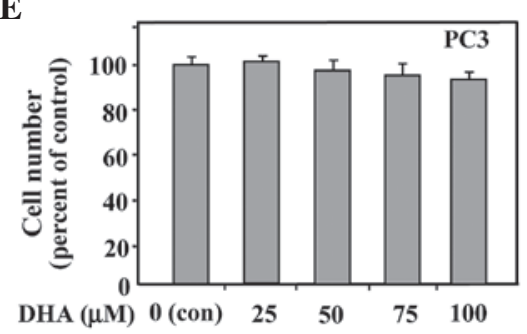

Figure 1. DHA exhibits no cytotoxic effect and inhibits hormone-dependent growth of LNCaP cells. (A) LNCaP cells cultured in medium containing 10\% FBS were treated with $100 \mu \mathrm{M}$ DHA for $6 \mathrm{~h}$ and subsequently trypan blue staining was performed. The cell numbers were counted to measure the viability. Ethanol treatment was used as a vehicle control. (B) LNCaP cells were assessed by an MTT assay for viability following exposure for $48 \mathrm{~h}$ to media containing $10 \% \mathrm{FBS}$ and varying concentrations of DHA. Equal quantities of ethanol were used as a vehicle control. (C) An MTT assay was performed on LNCaP cells following treatment with $50 \mu \mathrm{M}$ DHA for the indicated duration. (D and E) PC3 and DU145 cells growing in media containing 10\% FBS were treated with varying concentrations of DHA for $48 \mathrm{~h}$ and cell viability was measured using an MTT assay. The data are expressed as the mean \pm standard deviation for triplicate experiments. P-values were determined with Student's t-test. "P<0.01, compared with control. DHA, docosahexaenoic acid; MTT, 3, (4,5-dimethylthiazol-2-yl) 2,5-diphenyltetrazoliumbromide; con, control; FBS, fetal bovine serum 3, (4,5-dimethylthiazol-2-yl) 2,5-diphenyltetrazoliumbromide.

A

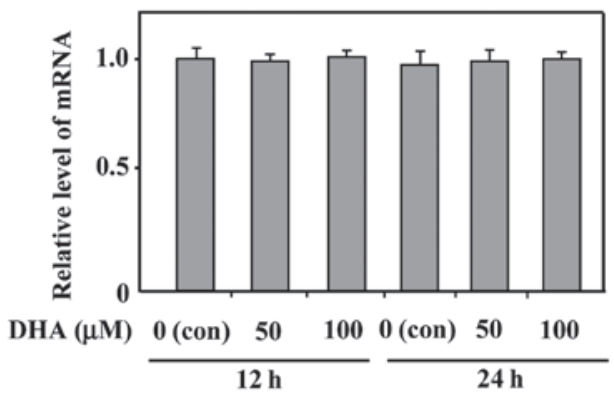

B

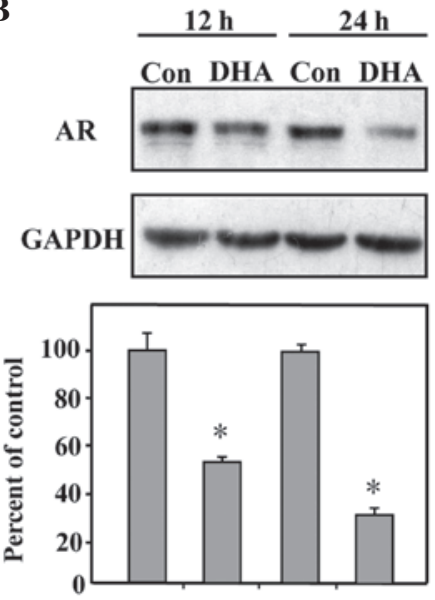

Figure 2. Effect of DHA on the expression of the AR. (A) The total RNA was extracted from LNCaP cells exposed for 12 or $24 \mathrm{~h}$ to media containing $10 \%$ fetal bovine serum and varying concentrations of DHA. The extracted RNA was subsequently used for reverse transcription-quantitative polymerase chain reaction. (B) Cell lysates from LNCaP cells treated with $50 \mu \mathrm{M}$ DHA were assessed by immunoblotting to determine the protein expression levels of the AR. GAPDH was used as internal control. The data are expressed as the mean \pm standard deviation for triplicate experiments. P-values were determined with Student's $t$-test. ${ }^{*} \mathrm{P}<0.01$, compared with control. AR, androgen receptor; DHA, docosahexaenoic acid; con, control.

DHA reduces the protein expression level of AR. To ascertain that DHA indeed affects the AR in LNCaP cells, the present study examined the effect of DHA on the expression levels of the AR. RT-qPCR analysis was performed to confirm whether the transcribed mRNA expression levels of the AR were affected by treatment with DHA. As shown in Fig. 2A, the mRNA expression levels of the AR in the DHA-treated LNCaP cells were unaltered compared with those from the 
control cells. The protein expression level of the AR was further assessed and the result of the immunoblotting revealed that treatment with $50 \mu \mathrm{M}$ DHA for 12 or $24 \mathrm{~h}$ downregulated the protein expression levels of the AR by 50 and $65 \%$, respectively (Fig. 2B). These data demonstrated that DHA exhibits no effect on the transcription of the AR gene, however, significantly reduces the protein expression level of the AR in LNCaP cells.

DHA represses androgen-regulated gene expression. Since androgen functions via the androgen receptor, which has been demonstrated to be reduced by DHA in LNCaP cells, the present study further investigated whether the androgen action was affected by DHA. RT-qPCR was performed to assess whether the mRNA expression level of androgen-responsive genes, including PSA, ODC, TMPRSS2, NKX3-1 and FKBP51, were affected by treatment with DHA. As shown in Fig. 3A, the mRNA expression levels of the selected genes were upregulated by androgen and treatment with $100 \mu \mathrm{M}$ DHA for $24 \mathrm{~h}$ significantly repressed the induced response. In addition, the quantity of secreted PSA was measured. The LNCaP cells cultured in serum-free media or exposed to R1881 were treated with different concentrations of DHA prior to the collection of the culture medium for measurement of the total protein expression levels of secreted PSA. As shown in Fig. 3B, androgen stimulated the expression of PSA and treatment with DHA decreased the androgen-induced expression of PSA in a dose-dependent manner. These data indicated that the actions of androgens can be inhibited in LNCaP cells by DHA.

$D H A$ promotes the proteasome-mediated degradation of $A R$. To further elucidate the discrepant effects of DHA on the mRNA and protein expression levels of the AR, the present study examined the effects of DHA on the protein expression of the AR at a range of durations. Treatment of the LNCaP cells with DHA revealed a time-dependent decrease in the protein expression level of the AR over the interval of 6-18 $\mathrm{h}$ (Fig. 4A). To ascertain whether this decline in AR protein level reflects a reduced protein synthesis or increased degradation by DHA, the protein translation inhibitor, $\mathrm{CHX}$, was used. Under conditions of $\mathrm{CHX}$ treatment and therefore, no protein translation, it was observed that the AR protein declined in a time-dependent manner, demonstrating a half-life of $12 \mathrm{~h}$ (Fig. 4B). Furthermore, the reduction in the AR protein was more pronounced with DHA in the presence of $\mathrm{CHX}$ (Fig. 4C). In addition, at the $18 \mathrm{~h}$ time point, there was a significant additive effect between DHA and CHX when LNCaP cells were treated with each drug (Fig. 4D). The additive reduction in the protein expression levels of the AR by addition of DHA beyond that already elicited by $\mathrm{CHX}$ indicated that the DHA-induced decrease in AR protein levels was not mediated by an inhibition of protein translation.

Since the evidence suggested that DHA has no effect on protein translation, however, reduces the protein level of AR, the present study next examined whether DHA acts as a regulator of AR stability. The LNCaP cells were treated with MG132, a proteasome inhibitor, to avoid proteasome-mediated degradation. As shown in Fig. 4E, treatment with MG132 increased the protein expression levels of the AR compared with the control, suggesting that $A R$ is degraded by the

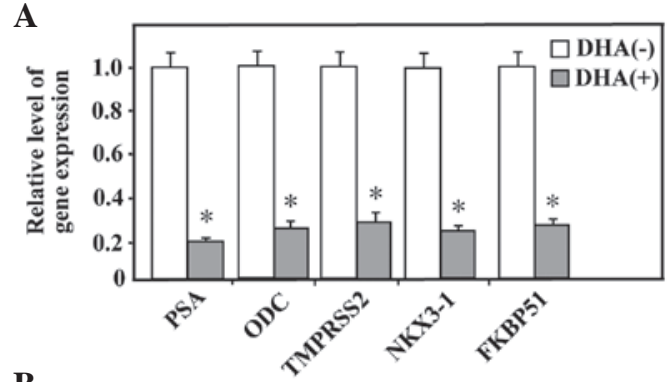

$\mathbf{B}$

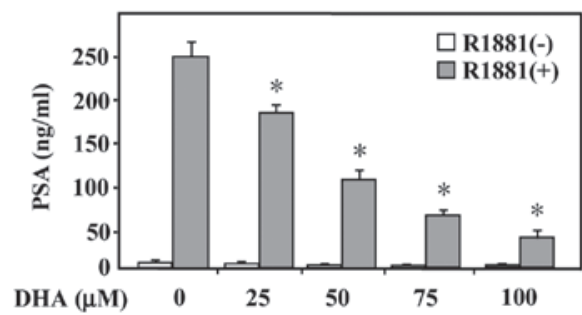

Figure 3. DHA represses androgen-regulated gene expression. (A) LNCaP cells growing in $10 \%$ complete fetal bovine serum medium were treated with or without $100 \mu \mathrm{M}$ DHA for $24 \mathrm{~h}$. The total RNA was extracted and used for reverse transcription-quantitative polymerase chain reaction analysis of the indicated genes. (B) The total PSA quantification was performed on the medium from LNCaP cells treated with varying concentrations of DHA in the absence or presence of $1 \mathrm{nM}$ R1881. The protein expression level of PSA was normalized to the 3, (4,5-dimethylthiazol-2-yl) 2,5-diphenyltetrazoliumbromide measurements. The data are expressed as the mean \pm standard deviation for triplicate experiments. P-values were determined with Student's t-test. ${ }^{*} \mathrm{P}<0.01$, compared with control. DHA, docosahexaenoic acid; con, control; PSA, prostate-specific antigen.

proteasome. Notably, the combined treatment of MG132 and DHA significantly increased the protein expression level of AR compared with treatment with DHA alone. The above data indicated that DHA promoted proteasome-mediated degradation of the AR.

\section{Discussion}

The incidence and mortality rate of prostate cancer differs among countries and regions. For instance, American males have a higher incidence and mortality of prostate cancer compared with Asian males (1). Several previous studies have indicated that dietary factors may be important in the incidence, progression and clinical outcome of prostate cancer (26-28). In addition, dietary fat has been demonstrated to promote or inhibit the growth of prostate cancer (29). Epidemiological and laboratory investigations have suggested that $\omega-3$ fatty acids inhibit the growth of prostate cancer cells and $\omega-6$ fatty acids promote the disease $(11,14,30,31)$. Based on this evidence, it has been speculated that the $\omega-3$ fatty acids may reduce the risk of prostate cancer and also inhibit the growth of developing prostate tumors.

It has been revealed that $\omega-3$ PUFAs repress the growth of prostate cancer cells in vitro and reduce the protein expression levels of the AR in LNCaP cells (24). However, the mechanism underlying the reduced protein expression level of the AR remains to be elucidated. The present study demonstrated for the first time, to the best of our knowledge, that DHA, a $\omega-3$ PUFA, promoted the degradation of the AR in LNCaP cells. 
A

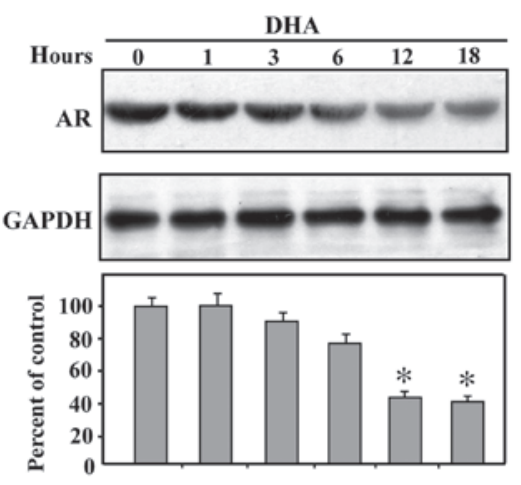

C
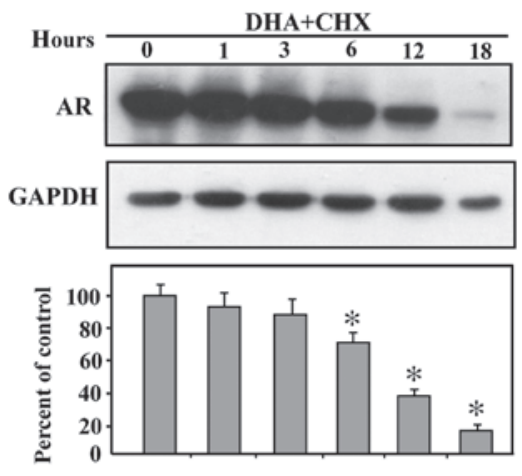

B

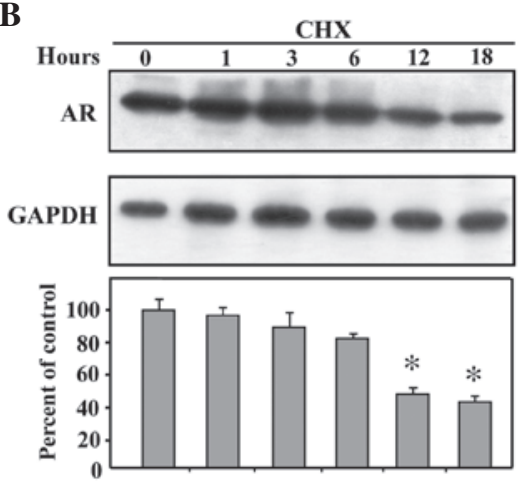

D

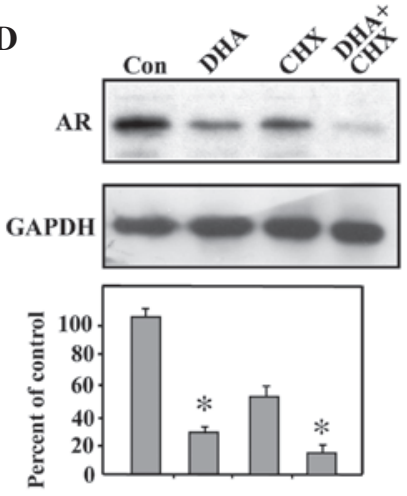

$\mathbf{E}$
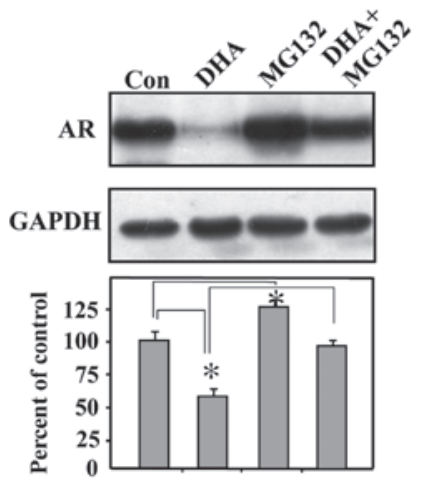

Figure 4. DHA promotes the degradation of the AR in LNCaP cells. (A) LNCaP cells cultured in medium containing $10 \%$ FBS were treated with $50 \mu \mathrm{M}$ DHA for the indicated durations. Cell lysates were prepared and used for immunoblotting and GAPDH was used as a loading control. (B) LNCaP cells cultured in medium containing 10\% FBS were treated with $50 \mu \mathrm{g} / \mathrm{ml} \mathrm{CHX}$ for the indicated durations. Cell lysates were prepared and used for immunoblotting and GAPDH was used as a loading control. (C) The LNCaP cells were treated with either DHA, CHX or both for $18 \mathrm{~h}$ and were subsequently lysed for immunoblotting. (D) The LNCaP cells treated with DHA combined with CHX for the indicated durations were lysed and used for immunoblotting. (E) The LNCaP cells were treated with DHA, MG132 or both for $24 \mathrm{~h}$. Cell lysates were prepared and used for immunoblotting. The data are expressed as the mean \pm standard deviation for triplicate experiments. P-values were determined with Student's t-test. "P<0.01, compared with contol. DHA, docosahexaenoic acid; con, control; FBS, fetal bovine serum; AR, androgen receptor; CHX, cyclohexamide.

Furthermore, androgenic induction of several androgen-regulated genes were significantly inhibited by DHA at steady-state mRNA expression levels. The above data indicated that DHA treatment repressed androgen action, including the cell growth response.

The present study also used EPA, another $\omega-3$ PUFA, to treat LNCaP cells, however, EPA has been demonstrated to have no significant repressive effect on LNCaP cell growth and revealed no reduction in the protein expression levels of the AR at concentrations $<100 \mu \mathrm{M}$. Although DHA and EPA are each long chain $\omega-3$ PUFAs, EPA contains less unsaturated bonds, which may result in a reduced inhibitory effect compared with DHA. It is also possible that the concentrations of EPA used were lower than required to exhibit its effect, since high concentrations of EPA have an inhibitory effect on the growth of LNCaP cells.

The present study demonstrated that DHA exhibits an inhibitory effect on the androgenic induction of gene expression. DHA inhibited the expression of the prostate-specific gene, PSA, and the ODC gene, which is ubiquitously expressed, which are well-known direct target genes of the AR. In addition, TMPRSS2, NKX3-1 and FKBP51, which are all upregulated by androgens, were also repressed by treatment with DHA. These results indicated that DHA can impair the transactivation ability of the AR. ODC is a rate-limiting enzyme in the polyamine biosynthesis pathway, which is known to be involved in the proliferation and differentiation of normal and neoplastic cells (32). Overexpression of ODC may 
be involved in the oncogenic process. Therefore, the repressed expression of ODC by DHA may partially explain the decrease in cell growth. The function of nuclear receptors, including the AR, can be affected by expression level. Androgens can stabilize the AR and therefore, increase the expression level of the AR. Immunoblot analysis of the AR demonstrated that DHA affected the androgen-mediated stabilizing effect by reducing the level of the AR.

It has been elucidated that one of the mechanisms by which prostate cancer cells become hormone-independent is by increasing the levels of the AR, thereby sensitizing the receptor to low levels of circulating androgens (33). Previous studies revealed that the hormone-independent LNCaP clones demonstrated a significant increase in the expression levels of the AR, as compared with their hormone dependent clones (24). Therefore, it may be helpful to reduce the levels of the AR during the progression to hormone-independency, to prevent the growth of $\mathrm{LNCaP}$ cells. The results from the present study demonstrated that treatment with DHA inhibited the upregulation of the AR, indicating that DHA may possibly be involved in modulating and regulating the AR pathway.

\section{Acknowledgements}

This study was supported by the Tianjin Municipal Science and Technology Commission (grant no. 12JCZDJC21600 to Dr Wei Hong), the National Natural Science Foundation of China (grant no. 81271203 to Dr Wei Hong), the German Academic Exchange Service DAAD (to Mr. Mohsen Esmaeili) and the German Cancer Aid (to Professor Aria Baniahmad) The authors would like to thank Dr David Fisher for critically reading the manuscript.

\section{References}

1. Saman DM, Lemieux AM, Nawal Lutfiyya M and Lipsky MS: A review of the current epidemiology and treatment options for prostate cancer. Dis Mon 60: 150-154, 2014.

2. Gathirua-Mwangi WG and Zhang J: Dietary factors and risk for advanced prostate cancer. Eur J Cancer Prev 23: 96-109, 2014.

3. Masko EM, Allott EH and Freedland SJ: The relationship between nutrition and prostate cancer: Is more always better? Eur Urol 63: 810-820, 2013.

4. Pelser C, Mondul AM, Hollenbeck AR and Park Y: Dietary fat, fatty acids and risk of prostate cancer in the NIH-AARP diet and health study. Cancer Epidemiol Biomarkers Prev 22: 697-707, 2013.

5. Wright JL, Plymate S, D'Oria-Cameron A, et al: A study of caloric restriction versus standard diet in overweight men with newly diagnosed prostate cancer: A randomized controlled trial. Prostate 73: 1345-1351, 2013.

6. Kang JX, Wan JB and He C: Concise review: Regulation of stem cell proliferation and differentiation by essential fatty acids and their metabolites. Stem Cells 32: 1092-1098, 2014.

7. Olivo SE and Hilakivi-Clarke L: Opposing effects of prepubertal low- and high-fat n-3 polyunsaturated fatty acid diets on rat mammary tumorigenesis. Carcinogenesis 26: 1563-1572, 2005.

8. Chen Z, Zhang Y, Jia C, et al: mTORC1/2 targeted by $\mathrm{n}-3$ polyunsaturated fatty acids in the prevention of mammary tumorigenesis and tumor progression. Oncogene 33: 4548-4557, 2014.

9. Apte SA, Cavazos DA, Whelan KA and Degraffenried LA: A low dietary ratio of omega- 6 to omega-3 Fatty acids may delay progression of prostate cancer. Nutr Cancer 65: 556-562, 2013.

10. Chua ME, Sio MC, Sorongon MC and Dy JS: Relationship of dietary intake of omega-3 and omega- 6 Fatty acids with risk of prostate cancer development: A meta-analysis of prospective studies and review of literature. Prostate Cancer 2012: 826254, 2012.
11. Akinsete JA, Ion G, Witte TR and Hardman WE: Consumption of high $\omega-3$ fatty acid diet suppressed prostate tumorigenesis in C3 (1) Tag mice. Carcinogenesis 33: 140-148, 2012.

12. Berquin IM, Edwards IJ and Chen YQ: Multi-targeted therapy of cancer by omega-3 fatty acids. Cancer Lett 269: 363-377, 2008.

13. Virtanen JK, Mozaffarian D, Chiuve SE and Rimm EB: Fish consumption and risk of major chronic disease in men. Am J Clin Nutr 88: 1618-1625, 2008.

14. Williams CD, Whitley BM, Hoyo C, et al: A high ratio of dietary $n-6 / n-3$ polyunsaturated fatty acids is associated with increased risk of prostate cancer. Nutr Res 31: 1-8, 2011.

15. Brasky TM, Till C, White E, et al: Serum phospholipid fatty acids and prostate cancer risk: Results from the prostate cancer prevention trial. Am J Epidemiol 173: 1429-1439, 2011.

16. Comba A, Lin YH, Eynard AR, Valentich MA, Fernandez-Zapico ME and Pasqualini ME: Basic aspects of tumor cell fatty acid-regulated signaling and transcription factors. Cancer Metastasis Rev 30: 325-342, 2011.

17. Stillwell W, Shaikh SR, Zerouga M, Siddiqui R and Wassall SR: Docosahexaenoic acid affects cell signaling by altering lipid rafts. Reprod Nutr Dev 45: 559-579, 2005.

18. Bazan NG: Omega-3 fatty acids, pro-inflammatory signaling and neuroprotection. Curr Opin Clin Nutr Metab Care 10: 136-141, 2007.

19. Balk SP: Androgen receptor functions in prostate cancer development and progression. Asian J Androl 16: 561-564, 2014.

20. Taplin ME and Balk SP: Androgen receptor: A key molecule in the progression of prostate cancer to hormone independence. J Cell Biochem 91: 483-490, 2004.

21. Wang LG, Ossowski L and Ferrari AC: Androgen receptor level controlled by a suppressor complex lost in an androgenindependent prostate cancer cell line. Oncogene 23: 5175-5184, 2004.

22. Chen CD, Welsbie DS, Tran C, Baek SH, Chen R, Vessella R, Rosenfeld MG and Sawyers CL: Molecular determinants of resistance to antiandrogen therapy. Nat Med 10: 33-39, 2004.

23. Linja MJ, Savinainen KJ, Saramäki OR, Tammela TL, Vessella RL and Visakorpi T: Amplification and overexpression of androgen receptor gene in hormone-refractory prostate cancer. Cancer Res 61: 3550-3555, 2001.

24. Friedrichs W, Ruparel SB, Marciniak RA and deGraffenried L: Omega-3 fatty acid inhibition of prostate cancer progression to hormone independence is associated with suppression of mTOR signaling and androgen receptor expression. Nutr Cancer 63: 771-777, 2011.

25. Hong W, Li J, Wang B, et al: Epigenetic involvement of Alien/ESET complex in thyroid hormone-mediated repression of E2F1 gene expression and cell proliferation. Biochem Biophys Res Commun 415: 650-655, 2011.

26. Joshi AD, John EM, Koo J, Ingles SA and Stern MC: Fish intake, cooking practices, and risk of prostate cancer: Results from a multi-ethnic case-control study. Cancer Causes Control 23: 405-420, 2012.

27. Huang M, Narita S, Numakura K, Tsuruta H, Saito M, Inoue T, Horikawa Y, Tsuchiya N and Habuchi T: A high-fat diet enhances proliferation of prostate cancer cells and activates MCP-1/CCR2 signaling. Prostate 72: 1779-1788, 2012.

28. Chang SN, Han J, Abdelkader TS, Kim TH, Lee JM, Song J, Kim KS, Park JH and Park JH: High animal fat intake enhances prostate cancer progression and reduces glutathione peroxidase 3 expression in early stages of TRAMP mice. Prostate 74: 1266-1277, 2014.

29. Berkow SE, Barnard ND, Saxe GA and Ankerberg-Nobis T: Diet and survival after prostate cancer diagnosis. Nutr Rev 65: 391-403, 2007.

30. Astorg P: Dietary N-6 and N-3 polyunsaturated fatty acids and prostate cancer risk: a review of epidemiological and experimental evidence. Cancer Causes Control 15: 367-386, 2004.

31. McEntee MF, Ziegler C, Reel D, et al: Dietary n-3 polyunsaturated fatty acids enhance hormone ablation therapy in androgen-dependentprostate cancer. Am J Pathol 173:229-241, 2008.

32. Symes AJ, Eilertsen M, Millar M, et al: Quantitative analysis of BTF3, HINT1, NDRG1 and ODC1 protein over-expression in human prostate cancer tissue. PLoS One 8: e84295, 2013.

33. Chen Y, Sawyers CL and Scher HI: Targeting the androgen receptor pathway in prostate cancer. Curr Opin Pharmacol 8: 440-448, 2008. 